\title{
Dimensional and temperature crossover in trapped Bose gases
}

\author{
U. Al Khawaja, ${ }^{1, *}$ N. P. Proukakis, ${ }^{2}$ J. O. Andersen, ${ }^{1, \dagger}$ M. W. J. Romans, ${ }^{1}$ and H. T. C. Stoof ${ }^{1}$ \\ ${ }^{1}$ Institute for Theoretical Physics, University of Utrecht, Leuvenlaan 4, 3584 CE Utrecht, The Netherlands \\ ${ }^{2}$ Department of Physics, University of Durham, South Road, Durham DH1 3LE, United Kingdom
}

(Received 22 March 2003; published 6 October 2003)

\begin{abstract}
We investigate the long-range phase coherence of homogeneous and trapped Bose gases as a function of the geometry of the trap, the temperature, and the mean-field interactions in the weakly interacting limit. We explicitly take into account the (quasi)condensate depletion due to quantum and thermal fluctuations, i.e., we include the effects of both phase and density fluctuations. In particular, we determine the phase diagram of the gas by calculating the off-diagonal one-particle density matrix and discuss the various crossovers that occur in this phase diagram and the feasibility of their experimental observation in trapped Bose gases.

DOI: 10.1103/PhysRevA.68.043603

PACS number(s): 03.75.Hh, 67.40.-w, 32.80.Pj
\end{abstract}

\section{INTRODUCTION}

The weakly interacting Bose gas in three dimensions has been studied in great detail over the past 50 years [1]. Below a critical temperature $T_{c} \simeq\left(2 \pi \hbar^{2} / m k_{B}\right)[n / \zeta(3 / 2)]^{2 / 3}$, where $n$ is the total density, the gas is in a Bose-Einstein condensed state. As with almost all thermodynamic phase transitions in three dimensions, a Bose-Einstein condensate leads to longrange order in the system. In this particular case, the longrange order is determined by the behavior of the off-diagonal one-particle density matrix $\left\langle\psi^{\dagger}(\mathbf{x}) \psi(\mathbf{0})\right\rangle$. If the one-particle density matrix goes to a constant $n_{c}$ in the limit $|\mathbf{x}| \rightarrow \infty$, a Bose-Einstein condensate is present and $n_{c}$ is the condensate density.

The physics of one- and two-dimensional Bose gases is very different from that of the three-dimensional one, which makes these low-dimensional systems very interesting. From a theoretical point of view, this difference is caused by the enhanced importance of the phase fluctuations [2-5]. In fact, the phase fluctuations are so large that in a homogeneous one-dimensional Bose gas at all temperatures and in a homogeneous two-dimensional Bose gas at any nonzero temperature, Bose-Einstein condensation cannot take place. This is the content of the Mermin-Wagner-Hohenberg theorem [6,7].

A natural question then arises: What happens if we have a large three-dimensional box with a Bose-Einstein condensed gas and squeeze two of the sides so as to obtain a onedimensional system? Similarly, what happens if we take the same box containing a Bose-Einstein condensed gas at a nonzero temperature and squeeze one of the sides so that a two-dimensional system results? These are questions that we would like to address in the present paper. Until recently, these questions could not be discussed on the basis of a microscopic theory, since no accurate equation of state existed for one- and two-dimensional Bose gases. However, we have recently developed an improved many-body $T$-matrix theory for partially Bose-Einstein condensed atomic gases by treat-

\footnotetext{
*Present address: Physics Department, United Arab Emirates University, P.O. Box 17551, Al-Ain, United Arab Emirates.

${ }^{\dagger}$ Present address: Nordita, Blegdamsvej 17, 2100 Copenhagen, Denmark.
}

ing the phase fluctuations exactly $[8,9]$. This mean-field theory is valid in arbitrary dimensions and accounts also for the (quasi)condensate depletion. It is therefore very suitable for describing the crossovers mentioned above.

Low-dimensional Bose gases are presently receiving a large amount of attention due to the recent experimental realization of one- and two-dimensional condensates in traps $[10,11]$ as well as one-dimensional gases on microchips $[12-$ 14]. Because these experiments deal with trapped, and therefore inhomogeneous, Bose gases it is important to consider this situation as well. The Mermin-Wagner-Hohenberg theorem $[6,7]$ is valid only in the thermodynamic limit, and so does not immediately apply to trapped Bose gases. In the trapped case, it turns out that under certain conditions the phase is coherent over a distance of the order of the size of the system and a "true" condensate is present. Under other conditions, the phase is coherent over a distance less than the size of the system and only a so-called "quasicondensate" [15] is present [2-5]. In fact, this situation can even occur in elongated three-dimensional Bose gases [16-21]. As a result, similar questions to those above arise: What happens to the phase fluctuations in a one-dimensional trapped Bose gas as we vary the temperature and the trap frequency? This is another question that we consider here.

The paper is organized as follows. In Sec. II, we briefly discuss the modified Popov theory presented in Refs. [8,9]. In Sec. III, we discuss dimensional crossovers and finite-size effects in a homogeneous Bose gas. In Sec. IV, the temperature crossover in both homogeneous and trapped Bose gases in one dimension is examined. Finally, we summarize and conclude in Sec. V.

\section{MODIFIED POPOV THEORY}

It is well known that the usual Popov theory for partially Bose-Einstein condensed gases suffers from infrared divergences in the equation of state at all temperatures in one dimension and at any nonzero temperature in two dimensions. These infrared problems arise because only quadratic fluctuations around the mean field have been taken into account. Specifically, the annihilation operator for the field is written as $\hat{\psi}(\mathbf{x})=\sqrt{n_{0}}+\hat{\psi}^{\prime}(\mathbf{x})$ and terms in the Hamiltonian that are of third order or higher in the fluctuating field $\hat{\psi}^{\prime}(\mathbf{x})$ 
are neglected. In Refs. $[8,9]$, it was shown that the problem of infrared divergences in the equation of state can be solved by taking into account phase fluctuations exactly and not only up to second order. The quadratic contribution to the density from the phase fluctuations is $n_{0}\langle\hat{\chi}(\mathbf{x}) \hat{\chi}(\mathbf{x})\rangle$, while an exact result would give no contribution at all. This follows from the identity $n_{0}\left\langle e^{-i \hat{\chi}(\mathbf{x})} e^{i \hat{\chi}(\mathbf{x})}\right\rangle=n_{0}[1$ $+\langle\hat{\chi}(\mathbf{x}) \hat{\chi}(\mathbf{x})\rangle+\cdots]=1$. In order to obtain the correct result, we must therefore subtract the quadratic contribution from the phase fluctuations. This ultimately results in the following expressions for the density $n$ and chemical potential $\mu$ :

$$
\begin{gathered}
n=n_{0}+\frac{1}{V} \sum_{\mathbf{k}}\left[\frac{\epsilon_{\mathbf{k}}-\hbar \omega_{\mathbf{k}}}{2 \hbar \omega_{\mathbf{k}}}+\frac{n_{0} T^{2 \mathrm{~B}}(-2 \mu)}{2 \epsilon_{\mathbf{k}}+2 \mu}+\frac{\epsilon_{\mathbf{k}}}{\hbar \omega_{\mathbf{k}}} N\left(\hbar \omega_{\mathbf{k}}\right)\right], \\
\mu=\left(2 n-n_{0}\right) T^{2 \mathrm{~B}}(-2 \mu)=\left(2 n^{\prime}+n_{0}\right) T^{2 \mathrm{~B}}(-2 \mu),
\end{gathered}
$$

where $n_{0}$ is the (quasi)condensate density and $n^{\prime}=n-n_{0}$ represents the depletion of the (quasi)condensate due to quantum and thermal fluctuations. It is important to point out that this theory takes the (quasi)condensate depletion into account, and can thus work even in the limit of $n_{0} / n \ll 1$, as will become apparent in subsequent sections. The Bogoliubov quasiparticle dispersion relation is given by $\hbar \omega_{\mathbf{k}}=\left[\epsilon_{\mathbf{k}}^{2}\right.$ $\left.+2 n_{0} T^{2 \mathrm{~B}}(-2 \mu) \epsilon_{\mathbf{k}}\right]^{1 / 2}$, where $\epsilon_{\mathbf{k}}=\hbar^{2} \mathbf{k}^{2} / 2 m$. Note also that the energy argument of the $T$ matrix is $-2 \mu$, because this is precisely the energy it costs to excite two atoms from the condensate $[22,23]$.

The Bose distribution function is given by $N(\varepsilon)$ $=1 /\left[\exp \left(\varepsilon / k_{B} T\right)-1\right]$, where $k_{B}$ is the Boltzmann constant and $T$ is the temperature. In addition, $T^{2 \mathrm{~B}}$ is the two-body $T$ matrix and $V$ is the volume of the system. The two-body $T$ matrix takes into account successive two-particle scattering in the vacuum, but neglects many-body effects of the medium. These many-body effects can be taken into account by using the many-body $T$ matrix instead of the two-body $T$ matrix [9]. For simplicity we shall use the two-body $T$ matrix for most of this work. However, in Sec. IV B we will actually use the many-body $T$ matrix to make the calculations for the trapped case as accurate as possible.

It is important to realize that the momentum sum on the right-hand side of Eq. (1) includes the term with $\mathbf{k}=\mathbf{0}$. This is a result of the fact that if we include fluctuations we have that $\left\langle\hat{\psi}_{\mathbf{0}}^{\dagger} \hat{\psi}_{\mathbf{0}}\right\rangle$, where $\hat{\psi}_{\mathbf{0}}=\int d \mathbf{x}[\hat{\psi}(\mathbf{x}) / V]$, is not exactly equal to $n_{0}$. This difference is most important at nonzero temperatures, and by taking the limit $\mathbf{k} \rightarrow \mathbf{0}$ in Eq. (1), we find that the contribution from the zero-momentum state to the density equals $n_{0}+(1 / V)\left(k_{B} T / 2 n_{0} T^{2 B}\right)$. This result can be physically understood by realizing that $\left\langle\hat{\psi}_{0}^{\dagger} \hat{\psi}_{0}\right\rangle$ can be found by calculating the average of $\left|\psi_{0}\right|^{2}$ with a probability distribution that obeys

$$
P\left(\left|\psi_{0}\right|\right)=e^{-\left(V T^{2 B} / k_{B} T\right)\left[-n_{0}\left|\psi_{0}\right|^{2}+(1 / 2)\left|\psi_{0}\right|^{4}\right]} .
$$

To be consistent, the above calculation has to be performed in the Bogoliubov approximation as well.
We define the normalized first-order correlation function $g(\mathbf{x})$ by

$$
\left\langle\hat{\psi}^{\dagger}(\mathbf{x}) \hat{\psi}(\mathbf{0})\right\rangle=\sqrt{n_{0}(\mathbf{x}) n_{0}(\mathbf{0})} g(\mathbf{x}) .
$$

In the large- $|\mathbf{x}|$ limit, it thus takes the form

$$
g(\mathbf{x}) \simeq e^{-1 / 2\left\langle[\hat{\chi}(\mathbf{x})-\hat{\chi}(\mathbf{0})]^{2}\right\rangle} .
$$

For a homogeneous Bose gas, the phase fluctuations are determined by $[8,9]$

$$
\begin{aligned}
\left\langle[\hat{\chi}(\mathbf{x})-\hat{\chi}(\mathbf{0})]^{2}\right\rangle= & \frac{T^{2 \mathrm{~B}}(-2 \mu)}{V} \sum_{\mathbf{k}}\left[\frac{1}{\hbar \omega_{\mathbf{k}}}\left[1+2 N\left(\hbar \omega_{\mathbf{k}}\right)\right]\right. \\
& \left.-\frac{1}{\epsilon_{\mathbf{k}}+\mu}\right][1-\cos (\mathbf{k} \cdot \mathbf{x})]
\end{aligned}
$$

The analogous expression for the one-dimensional harmonically confined condensate has also been calculated. In the Thomas-Fermi limit and neglecting quantum fluctuations, it reads [9]

$$
\begin{aligned}
\langle[\hat{\chi}(z) & \left.-\hat{\chi}(0)]^{2}\right\rangle \\
= & \frac{4 \pi \kappa l_{z}^{4}}{R_{\mathrm{TF}}^{3}} \sum_{j=0} N\left(\hbar \omega_{j}\right)\left[A_{j}^{2}\left[P_{j}\left(z / R_{\mathrm{TF}}\right)-P_{j}(0)\right]^{2}\right. \\
& \left.-B_{j}^{2}\left(\frac{P_{j}\left(z / R_{\mathrm{TF}}\right)}{1-\left(z / R_{\mathrm{TF}}\right)^{2}}-P_{j}(0)\right)^{2}\right]
\end{aligned}
$$

where $A_{j}=\sqrt{(j+1 / 2) \mu^{\prime} / \hbar \omega_{j}}, B_{j}=\sqrt{(j+1 / 2) \hbar \omega_{j} / \mu^{\prime}}$, and $P_{j}(z)$ is the Legendre polynomial of order $j$. Here $\mu^{\prime}=\mu$ $-2 \kappa n^{\prime}(0), n^{\prime}(0)$ is depletion in the center of the trap, and the coupling constant $\kappa=a / 2 \pi \ell_{\perp}^{2}$ results from averaging the three-dimensional two-body $T$ matrix, which is equal to $4 \pi a \hbar^{2} / m$ with $a$ the $s$-wave scattering length, over the cross-sectional area of the one-dimensional condensate. Furthermore, $\ell_{\perp}$ is the harmonic oscillator length in the radial direction. In the axial direction, the trap length is $\ell_{z}$ $=\sqrt{\hbar / m \omega_{z}}$, where $\omega_{z}$ is the harmonic oscillator frequency in the $z$ direction. The quantity $R_{\mathrm{TF}}$ denotes the temperaturedependent Thomas-Fermi radius, defined by the point at which the quasicondensate density $n_{0}(z)=\left[\mu^{\prime}-V^{\text {trap }}(z)\right]$ vanishes. Here $V^{\text {trap }}(z)$ is the harmonic trapping potential in the axial direction. The frequencies $\omega_{j}$ are given by $\omega_{j}$ $=\sqrt{j(j+1) / 2} \omega_{z}$.

If the function $g(\mathbf{x})$ approaches a constant in the large- $|\mathbf{x}|$ limit, the system contains a true Bose-Einstein condensate. If $g(\mathbf{x})$ goes to zero algebraically in the limit $|\mathbf{x}| \rightarrow \infty$, the system is said to contain a quasicondensate. Finally, if $g(\mathbf{x})$ goes to zero exponentially fast, the system is in the normal state. In the following, we calculate the correlation function $g(\mathbf{x})$ for various situations. 
a)
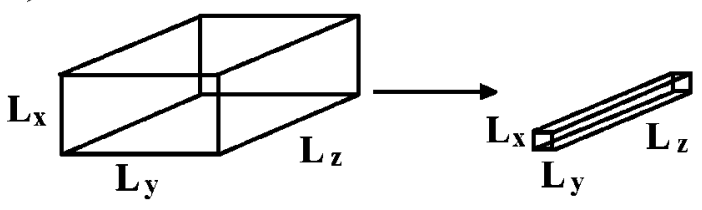

b)

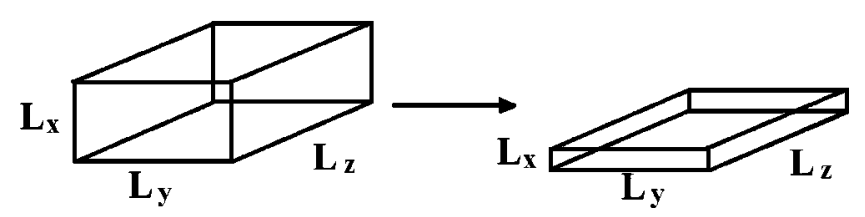

FIG. 1. Illustration of the geometry for (a) the dimensional crossover from a three-dimensional to a one-dimensional Bose gas, and (b) the dimensional crossover from a three-dimensional to a two-dimensional Bose gas.

\section{DIMENSIONAL CROSSOVER IN A HOMOGENEOUS BOSE GAS}

In this section, we investigate the dimensional crossover from three to one dimension at zero temperature, as well as the dimensional crossover from three to two dimensions at nonzero temperature. The relevant geometry is illustrated in Fig. 1.

\section{A. Crossover from three dimensions to one dimension at zero temperature}

We consider a uniform Bose gas in a box with lengths $L_{x}$, $L_{y}$, and $L_{z}$ and impose periodic boundary conditions. Due to the boundary conditions, the three components of the wave vector $\mathbf{k}$ take on discrete values, i.e., $k_{i}=2 \pi n_{i} / L_{i}$, where $i=x, y, z$ and $n_{i}$ is an integer. Substituting this into Eq. (6), we obtain

$$
\begin{aligned}
\left\langle[\hat{\chi}(z)-\hat{\chi}(0)]^{2}\right\rangle= & \frac{1}{2 N_{0}} \sum_{n_{x}, n_{y}, n_{z}}\left[\frac{1}{\sqrt{(k \xi)^{4}+(k \xi)^{2}}}\right. \\
& \left.-\frac{1}{(k \xi)^{2}+\frac{1}{2}}\right]\left[1-\cos \left(\frac{n_{z} z}{\lambda_{z} \xi}\right)\right],
\end{aligned}
$$

where $N_{0}$ is the number of atoms in the (quasi)condensate, $\lambda_{z}=L_{z} / 2 \pi \xi$, and $\xi=\hbar /\left[4 m n_{0} T^{2 \mathrm{~B}}(-2 \mu)\right]^{1 / 2}$ is the correlation length. Note that we have neglected the contribution from $n^{\prime}$ to the chemical potential, which is a good approximation in the weak-coupling limit we are considering. Note that the argument of the cosine has been simplified by choosing the direction of $\mathbf{x}$ to be along the $z$ axis.

For very elongated systems, one side of the box is much larger than the other two, and we choose $L_{z} \gg L_{x}, L_{y}$. If we also take $L_{z}$ to be much larger than the correlation length, we can approximate the sum over $n_{z}$ by an integral. In the limit $z / \xi \gg 1$, we obtain

$$
\begin{aligned}
& \left\langle[\hat{\chi}(z)-\hat{\chi}(0)]^{2}\right\rangle \\
& \quad=\frac{\lambda_{z}}{N_{0}}\left[\gamma-\frac{\pi}{\sqrt{2}}+\ln 2+\ln \left(\frac{z}{\xi}\right)\right.
\end{aligned}
$$

$$
\left.+\sum_{n_{x}, n_{y}}\left(\frac{K_{0}\left(-\frac{1}{t_{x, y}}\right)}{\sqrt{t_{x, y}}}-\frac{\pi}{2 \sqrt{t_{x, y}}+\frac{1}{2}}\right)\right] \text {, }
$$

where

$$
t_{x, y}=\left(\frac{n_{x}}{\lambda_{x}}\right)^{2}+\left(\frac{n_{y}}{\lambda_{y}}\right)^{2}
$$

and the prime on the sum indicates that the term where $n_{x}$ $=n_{y}=0$ is omitted. Here $K_{0}(z)$ is a modified Bessel function of the second kind, $\lambda_{x, y}=L_{x, y} / 2 \pi \xi$, and $\gamma \simeq 0.5772$ is Euler's constant.

The one-dimensional limit is obtained by letting $L_{x, y}$ $\rightarrow 0$. This is equivalent to keeping only the first term on the right-hand side of Eq. (9), thus

$$
\left\langle[\hat{\chi}(z)-\hat{\chi}(0)]^{2}\right\rangle=\frac{1}{2 \pi n_{0} \xi}\left[\gamma-\frac{\pi}{\sqrt{2}}+\ln 2+\ln \left(\frac{z}{\xi}\right)\right],
$$

where $n_{0}=N_{0} / L_{z}$ is the one-dimensional quasicondensate density. The result (11) shows that the correlation function $g(z)$ at large distances falls off algebraically with the exponent $\eta=1 / 4 \pi n_{0} \xi$. In the weakly interacting limit $4 \pi n \xi \gg 1$ the depletion is small, so that we indeed have that $n_{0} \simeq n$. Keeping this in mind, Eq. (11) is in complete agreement with the exact result obtained by Haldane [24].

The three-dimensional limit it obtained by letting $L_{x, y}$ $\rightarrow \infty$. The discrete sums in Eq. (9) then become integrals. Performing the integrations, we obtain in the limit $z / \xi \rightarrow \infty$

$$
\left\langle[\hat{\chi}(z)-\hat{\chi}(0)]^{2}\right\rangle=\frac{\sqrt{2} \pi-4}{16 \pi^{2} n_{0} \xi^{3}},
$$

where $n_{0}=N_{0} / L_{x} L_{y} L_{z}$ is now the three-dimensional quasicondensate density. Equation (12) shows that the correlation function in three dimensions goes to a constant, so we have a "true" condensate. Note, however, that in three dimensions the exponent in Eq. (5) does not vanish and therefore that $n_{0}$ appearing in Eqs. (1) and (2) is not exactly equal to the condensate density $n_{c}$. In fact, Eq. (12) shows that the condensate density $n_{c}$ is given by

$$
n_{c}=n_{0} \exp \left(\frac{4-\sqrt{2} \pi}{32 \pi^{2} n_{0} \xi^{3}}\right)=n\left[1-\frac{8}{3} \sqrt{\frac{n a^{3}}{\pi}}+O\left(n a^{3}\right)\right]
$$


where $n$ is the total density of the gas. This result is in full agreement with that of the Popov or Bogoliubov theory.

The dimensional crossover behavior can be investigated in detail from Eq. (9) by calculating the phase fluctuations $\left\langle[\hat{\chi}(z)-\hat{\chi}(0)]^{2}\right\rangle$ for different finite values of $\lambda_{x}$ and $\lambda_{y}$. Comparing Eqs. (11) and (12), it follows that the phase fluctuations scale with the product $\lambda_{x} \lambda_{y}$ in the three-dimensional limit. In order to plot the phase fluctuations for different sizes of the box, it is therefore convenient to multiply $\left\langle[\hat{\chi}(z)-\hat{\chi}(0)]^{2}\right\rangle$ by a scaling function $f\left(\lambda_{x}, \lambda_{y}\right)$ that goes to $1 / \lambda_{x} \lambda_{y}$ in the three-dimensional limit. Moreover, it follows from Eq. (11) that $f\left(\lambda_{x}, \lambda_{y}\right)$ must approach unity in the onedimensional limit. A simple choice for a scaling function that has these properties is

$$
f\left(\lambda_{x}, \lambda_{y}\right)=\frac{1}{\left(\lambda_{x}+1\right)\left(\lambda_{y}+1\right)}
$$

In the following, we take $\lambda_{x}=\lambda_{y}=\lambda$ for simplicity.

In Fig. 2, we plot $\left\langle[\hat{\chi}(z)-\hat{\chi}(0)]^{2}\right\rangle N_{0} /(2 \pi)^{3}(1+\lambda)^{2} \lambda_{z}$ as a function of $\ln (z / \xi)$ for three values of $\lambda$. In the threedimensional limit $\lambda \rightarrow \infty$, the phase fluctuations are independent of $z$ for large $z / \xi$. For finite values of $\lambda$, the curves deviate from the $\ln (z / \xi)$ behavior due to contributions from the sum in Eq. (9). It should be noted that convergence of the sum in the left-hand side of Eq. (8) depends on the value of $\lambda$. For large $\lambda$, we included more terms in the sum to obtain convergence. The summations were terminated when the error is less than $0.1 \%$ of the three-dimensional result.

\section{B. Crossover from three dimensions to two dimensions at a nonzero temperature}

The nonzero-temperature part of the phase fluctuations is given by

$$
\frac{1}{N_{0}} \sum_{n_{x}, n_{y}, n_{z}} \frac{1-\cos \left(\frac{n_{z} z}{\lambda_{z} \xi}\right)}{k \xi \sqrt{(k \xi)^{2}+1}\left(e^{2 n_{0} T^{2 \mathrm{~B}} k \xi \sqrt{(k \xi)^{2}+1} / k_{B} T}-1\right)} .
$$

The first step consists of taking the limit $L_{y}=L_{z} \rightarrow \infty$. The sums over $n_{y}$ and $n_{z}$ then become integrals. The twodimensional limit is obtained by letting $L_{x} \rightarrow 0$, which is equivalent to keeping only the $n_{x}=0$ term in the above sum. After going to polar coordinates, this gives

$$
\begin{aligned}
& \frac{\lambda^{2}}{N_{0}} \int_{0}^{\infty} \int_{0}^{2 \pi} \frac{d(k \xi) d \phi}{\sqrt{(k \xi)^{2}+1}\left(e^{2 n_{0} T^{2 \mathrm{~B}} k \xi \sqrt{(k \xi)^{2}+1} / k_{B} T}-1\right)} \\
& \times[1-\cos (k z \cos \phi)]
\end{aligned}
$$

with $\lambda_{y}=\lambda_{x}=\lambda$. Integration over $\phi$ leads to

$$
\frac{2 \pi \lambda^{2}}{N_{0}} \int_{0}^{\infty} d(k \xi) \frac{1-J_{0}(k z)}{\sqrt{(k \xi)^{2}+1}\left(e^{2 n_{0} T^{2 \mathrm{~B}} k \xi \sqrt{(k \xi)^{2}+1} / k_{B} T}-1\right)},
$$

where $J_{0}(z)$ is a Bessel function of the first kind. The integral behaves as $k_{B} T \ln (z / \xi) / 2 n_{0} T^{2 \mathrm{~B}}$ in the limit $z / \xi \gg 1$. Thus the first-order correlation function obeys

$$
g(z) \simeq \frac{1}{(z / \xi)^{1 / n_{0} \Lambda^{2}}}
$$

where $\Lambda=\sqrt{2 \pi \hbar^{2} / m k_{B} T}$ is the thermal de Broglie wavelength.

The three-dimensional limit is obtained by letting $L_{x}$ $=L_{y}=L_{z} \rightarrow \infty$ in Eq. (15). The sums over $n_{x}, n_{y}$, and $n_{z}$ then become integrals. Going to polar coordinates and integrating over angles, we obtain

$$
\begin{aligned}
& \frac{2 \pi \lambda^{3}}{N_{0}} \int_{0}^{\infty} \frac{d(k \xi) k \xi}{\sqrt{(k \xi)^{2}+1}} \frac{1}{\left(e^{2 n_{0} T^{2 \mathrm{~B}} k \xi \sqrt{(k \xi)^{2}+1} / k_{B} T}-1\right)} \\
& \quad \times\left[1-\frac{\sin (k z)}{k z}\right] .
\end{aligned}
$$

The first term is a constant, independent of $z$. The second term is oscillating and goes to zero in the limit $z / \xi \rightarrow \infty$. This shows that the total integral, and thus the phase fluctuations, go to a nonzero constant and that Bose-Einstein condensation is possible in three dimensions at nonzero temperature. For low temperatures, where the phonons give the main contribution to the above integral, this constant is proportional to $1 / n_{0} \xi \Lambda^{2} \ll 1$. Combining, the above result we see that the crossover behavior is therefore qualitatively very similar to the one presented in Fig. 2.

\section{TEMPERATURE CROSSOVER IN A FINITE ONE-DIMENSIONAL BOSE GAS}

In this section, we first study the temperature crossover in a homogeneous one-dimensional Bose gas. The aim is to capture the essential crossover physics and to find the relevant parameters. Having established the relevant parameters, we examine a harmonically trapped one-dimensional Bose gas.

\section{A. Homogeneous Bose gas}

In this subsection we calculate the nonzero-temperature correlation function of a homogeneous Bose gas in one dimension. We construct the phase diagram by plotting this function versus the temperature $T$ and the length $L$ of the system. In detail the procedure is as follows.

We first solve the two coupled Eqs. (1) and (2) for the (quasi)condensate density $n_{0}$ at a fixed temperature, system size $L$, and for a total density $n=N / L$, where $N$ is the fixed total number of atoms. Then we use this solution in Eq. (6) to calculate the correlation function. To obtain a universal phase diagram, we need to scale $T$ and $L$ to some characteristic 


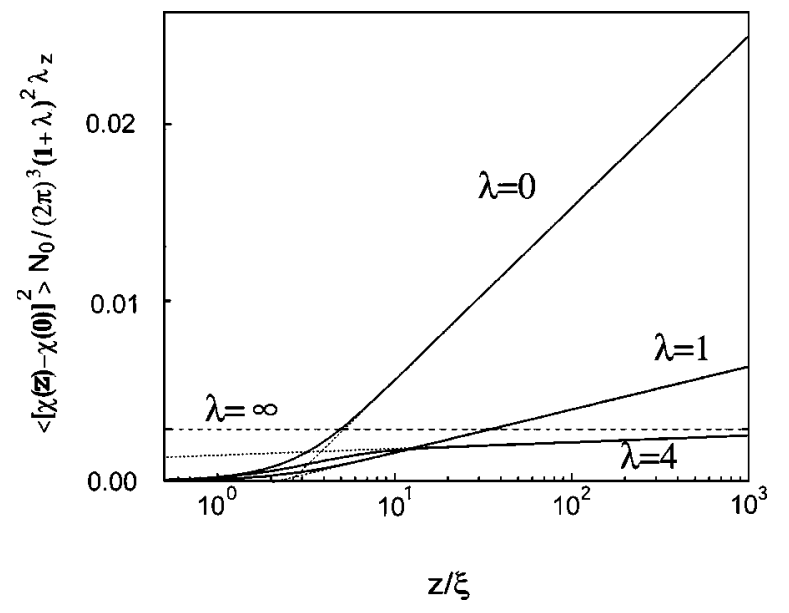

FIG. 2. Zero-temperature crossover behavior in the phase fluctuations of a three-dimensional homogeneous Bose-Einstein condensate. The three full lines show the phase fluctuations $\langle[\hat{\chi}(z)$ $\left.-\hat{\chi}(0)]^{2}\right\rangle N_{0} /(2 \pi)^{3}(1+\lambda)^{2} \lambda_{z}$ as a function of $\ln (z / \xi)$, for three values of $\lambda$. The dotted lines are calculated from Eq. (9).

temperature and length in the system. In the present case our homogeneous system is characterized by its correlation length $\xi$. The correlation length is normally defined in terms of the condensate density. However, above the Bose-Einstein transition temperature, the normal state cannot be characterized by this length. Therefore we define the correlation length in terms of the total density, rather than the condensate density, namely $\xi=1 / \sqrt{8 \pi \kappa n}$. As a result, we scale lengths to $\xi$ and temperature to $T_{0}=\hbar^{2} / 8 \pi m \xi^{2}$. The scaled temperature $T / T_{0}$ is equivalent to $(\xi / \Lambda)^{2}$, where $\Lambda$ $=\sqrt{2 \pi \hbar^{2} / m k_{\mathrm{B}} T}$ is the de Broglie wavelength.

In Fig. 3 we plot the correlation function at $z=0.9 L$ versus the dimensionless variables $L / \xi$ and $(\xi / \Lambda)^{2}$ for a fixed number of atoms. This displays clearly the crossover from a condensate near the origin and near the axes, where $g \simeq 1$, to a quasicondensate in the regions away from the origin and

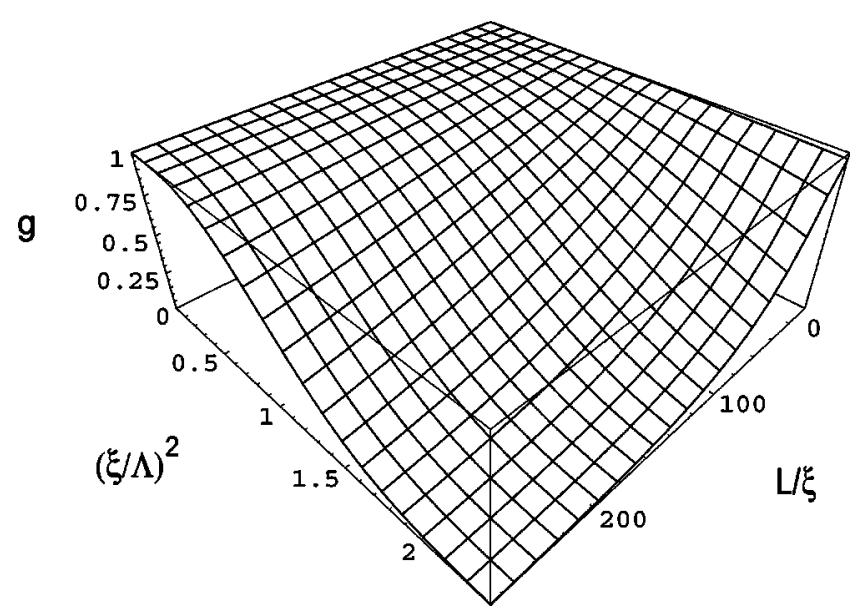

FIG. 3. The correlation function of a homogeneous onedimensional condensate as a function of $L / \xi$ and $(\xi / \Lambda)^{2}$. The correlation function is calculated at $z=0.9 L$ and for a total number of atoms $N=2000$.

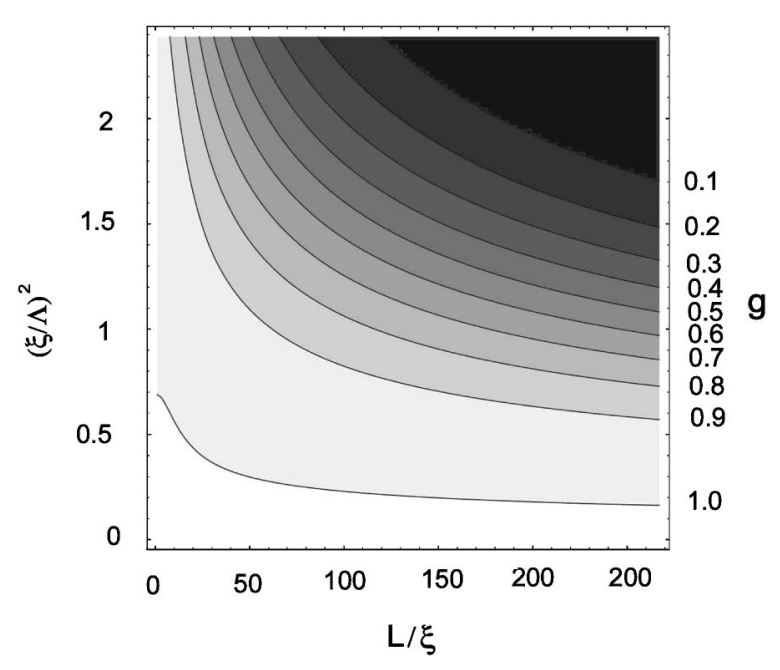

FIG. 4. The contour plot corresponding to Fig. 3. The value of $g$ along each contour line is indicated on the right vertical axis.

the axes. Figure 4 is a two-dimensional contour plot that is equivalent to Fig. 3 . The contour lines in this figure show the relation between $L / \xi$ and $(\xi / \Lambda)^{2}$ for a fixed value of $g$. Note that when quantum fluctuations can be neglected, we expect that $-\ln [g(0.9 L)] \propto\left(L / N_{0} \xi\right)(\xi / \Lambda)^{2}(0.9 L / \xi)$, which shows that for a fixed value of $g$ we have that $(\xi / \Lambda)^{2} \propto(L / \xi)^{-2}$. This relation is indeed borne out by a detailed inspection of Fig. 4. The theory discussed in this paper is only valid in the weakly interacting limit $4 \pi n \xi \gg 1$ and therefore does not include the Tonks regime $[25,26]$. Nonetheless, it is instructive to indicate at what region of the phase diagram such a regime may exist. The criterion for the Tonks gas limit is $N<4 \pi \kappa L[5,27]$. Upon using the above-mentioned scaling, this limit reads $L / \xi>\sqrt{2} N$. Since Fig. 3 was generated with $N=2000$, it is clear that in order to be in the Tonks gas limit, the condition $L / \xi>2800$ must be satisfied.

\section{B. Trapped Bose gas}

Next, we construct the phase diagram of a onedimensional Bose gas confined by a harmonic potential in order to investigate the effect of the induced density inhomogeneity. Our treatment is based on the local-density approximation for the calculation of the density profile, and is performed within the many-body $T$-matrix approximation for the interatomic interactions (as opposed to the two-body limit used in the preceding homogeneous calculations). The calculation of the correlation function is again performed for a fixed number of atoms, but using now Eq. (7). In the trapped case, a measure of the size of the system can be determined by the Thomas-Fermi radius $R_{\mathrm{TF}}$, which is, in general, a function of both temperature and trapping frequency. To plot a universal phase diagram, however, the axis corresponding to the system size should be independent of the other axis representing the temperature. This argument is strengthened by the fact that the temperature-dependent Thomas-Fermi radius $R_{\mathrm{TF}}(T)$ does not increase monotonically with increasing confinement at fixed temperature and total number of atoms. Such an effect is a direct consequence of two competing mechanisms which have a different depen- 


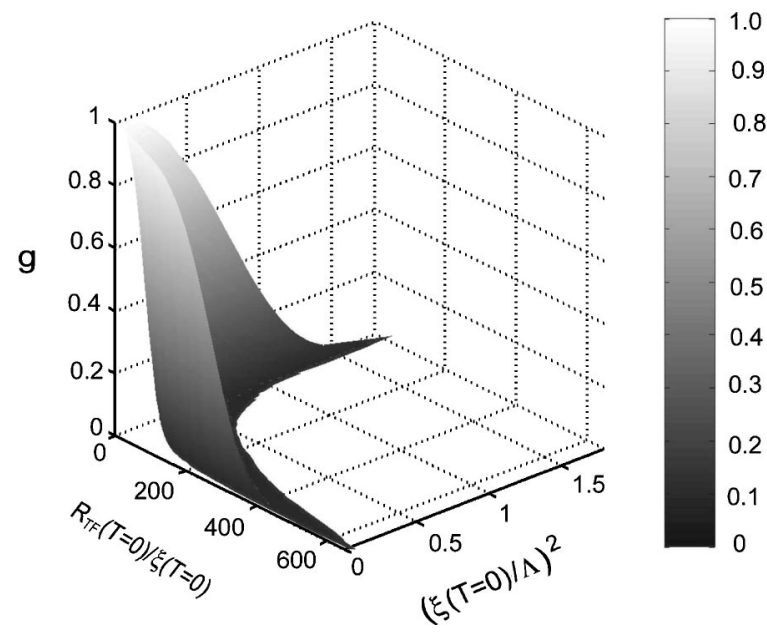

FIG. 5. Universal phase diagram of a pure one-dimensional trapped atomic Bose gas with constant number of atoms. Gray scale indicates the value of $g\left(0, z=0.9 R_{\mathrm{TF}}(T)\right)$ with lighter regions corresponding to maximum coherence $g \simeq 1$. This figure probes the entire region from the normal-to-(quasi)condensate crossover [where the (quasi)condensate depletion approaches $100 \%$, curved region away from origin] to the presence of true Bose-Einstein condensation, for which the quasicondensate depletion becomes negligible near the origin. The entire phase diagram plotted here remains well within the validity of our Thomas-Fermi approximation, with $\gamma=N_{0} \kappa l_{z} \gg 1$ and $N_{0}$ the number of (quasi)condensate atoms. For a ${ }^{23} \mathrm{Na}$ condensate with $N=2000$ atoms under very tight transverse confinement of $\omega_{\perp} / 2 \pi=5 \mathrm{kHz}$, the above phase diagram would correspond to temperatures in the range $0.2 \mathrm{nK}-12 \mu \mathrm{K}$ and longitudinal frequencies $\omega_{z} / 2 \pi$ from 0.05 to $45000 \mathrm{~Hz}$.

dence on the confinement: As the trap frequency increases, one would at first expect the size of the (quasi)condensate to decrease monotonically. However, due to the constraint on the total number of atoms, the number of atoms in the (quasi)condensate tends to increase simultaneously. This can lead to an increase in $R_{\mathrm{TF}}(T)$, in particular at higher temperatures.

To obtain a universal phase diagram in the case of a fixed total number of atoms, we hence use the zero-temperature Thomas-Fermi radius $R_{\mathrm{TF}}(T=0)$ as a measure of the size of the system. This is again scaled to the zero-temperature correlation length $\xi(T=0)$. The latter is evaluated in terms of the central density $n(0)$ via $\xi(T=0)=1 / \sqrt{8 \pi \kappa n(0)}$ $=\hbar / \sqrt{2 \pi \mu m}$ where the one-dimensional chemical potential at zero temperature in the Thomas-Fermi approximation is given by $\mu=(3 \pi / \sqrt{2})^{2 / 3}\left(N \kappa l_{z}\right)^{2 / 3} \hbar \omega_{z}$ [9]. Note that the actual size of the (quasi)condensate is given by the temperature-dependent $R_{\mathrm{TF}}(T)$, so a good measure of the macroscopic coherence of the system is obtained by measuring the correlation function at $0.9 R_{\mathrm{TF}}(T)$ [9].

Figure 5 shows the value of the normalized correlation function of Eq. (5) evaluated at $z=0.9 R_{\mathrm{TF}}(T)$ as a function of $R_{\mathrm{TF}}(T=0) / \xi(T=0)$ and $[\xi(T=0) / \Lambda]^{2}$ for fixed coupling constant $\kappa$ and total number of atoms $N$ in the system. Light color indicates the presence of a pure condensate and black color indicates the absence of universal phase coherence across the system. As expected, the coherence is found to increase when decreasing the size of the system, i.e., increasing the trap frequency $\omega_{z}$ at fixed temperature, or decreasing the temperature in a fixed trap. This is similar to the homogeneous case, with $g$ becoming close to 1 as $R_{\mathrm{TF}}(T$ $=0) / \xi(T=0)$ or $[\xi(T=0) / \Lambda]^{2}$ becomes small. Note that the quasicondensate depletion due to interactions and nonzero temperatures can be very significant, and this figure covers the entire range of (quasi)condensate fractions, ranging from 1 at the origin, where we essentially deal with a pure condensate, to approximately zero near the transition to the normal state sufficiently far from the origin. In particular, the characteristic curved shape away from the origin marks a sharp transition from a quasicondensate to the normal phase. Note that we find a sharp transition due to the use of the local-density approximation, whereas in reality this would be a smooth crossover. To study the crossover between regions of different degree of coherence within the (quasi)condensate regime, Fig. 6(a) shows the corresponding contour plot, focusing on a small region near the origin where the crossover takes place. In this figure, white regions correspond to true Bose-Einstein condensation whereas the darkest region on the top right corner of the figure corresponds to a complete absence of phase coherence across the size of the system. The corresponding contour lines with both axes plotted on a logarithmic scale are shown in Fig. 6(b).

The above phase diagram corresponds to the limit of a pure 1D Bose gas. In trying to probe this experimentally by means of the recently produced quasi-1D Bose gases [1014], there are certain constraints which must be taken into consideration. First, the temperature must be low enough that transverse thermal excitations are suppressed, i.e., $k_{B} T$ $<\hbar \omega_{\perp}$. Second, the chemical potential must not exceed the transverse energy, i.e., $\mu<\hbar \omega_{\perp}$, thus restricting the maximum longitudinal frequencies for which the system remains kinematically one-dimensional to some fraction of the transverse confinement. The exact fraction depends on the atomic species, the scattering length, the number of atoms, and the temperature. Finally, the longitudinal confinement cannot be made arbitrarily weak, as it will then be essentially impossible to trap the atoms. All the above constraints require a very large transverse confinement, since the experimentally accessible region of such a phase diagram increases with increasing transverse confinement. The experimental constraint of minimum longitudinal frequency does not pose much of a limitation since the low-frequency regions correspond to the outermost points of Fig. 5, for which the macroscopic coherence has already been completely lost. Of the other two constraints, which is more restrictive depends on the details of the particular experiment, since they exclude different regions of the phase diagram.

To illustrate this, Fig. 7(a) indicates the experimentally accessible regions for the case of $N=2000{ }^{23} \mathrm{Na}$ condensate atoms under a transverse confinement of $\omega_{\perp} / 2 \pi=5 \mathrm{KHz}$ [28]. The chemical potential constraint generally excludes a region to the left of Fig. 6(a), and so large coherences are best probed by restricting the condensate to a very small number of atoms. For the case studied here, this translates to the approximate condition $R_{\mathrm{TF}}(T=0) / \xi(T=0)>40$. The temperature constraint, on the other hand, prohibits a large 


\section{(a)}

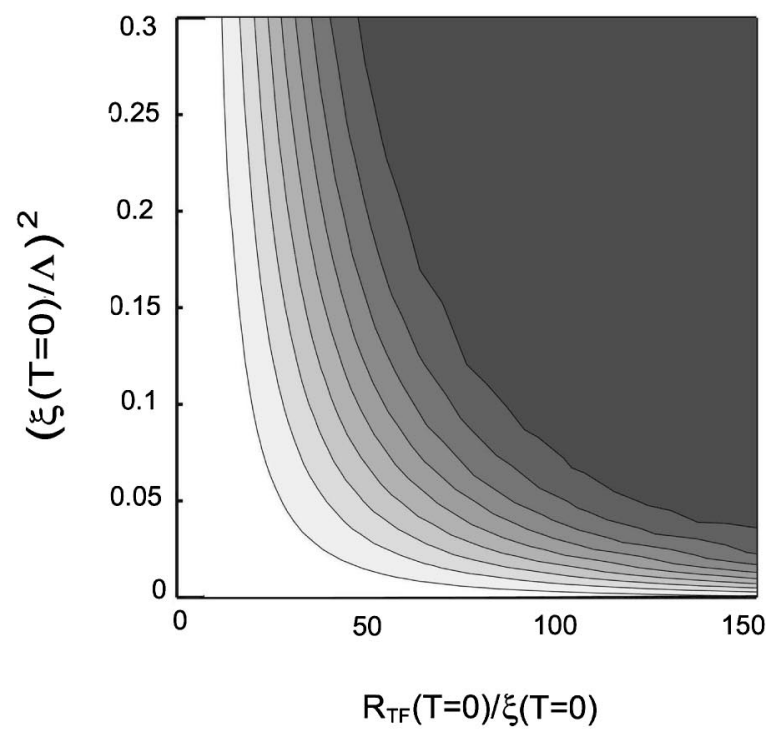

(b)

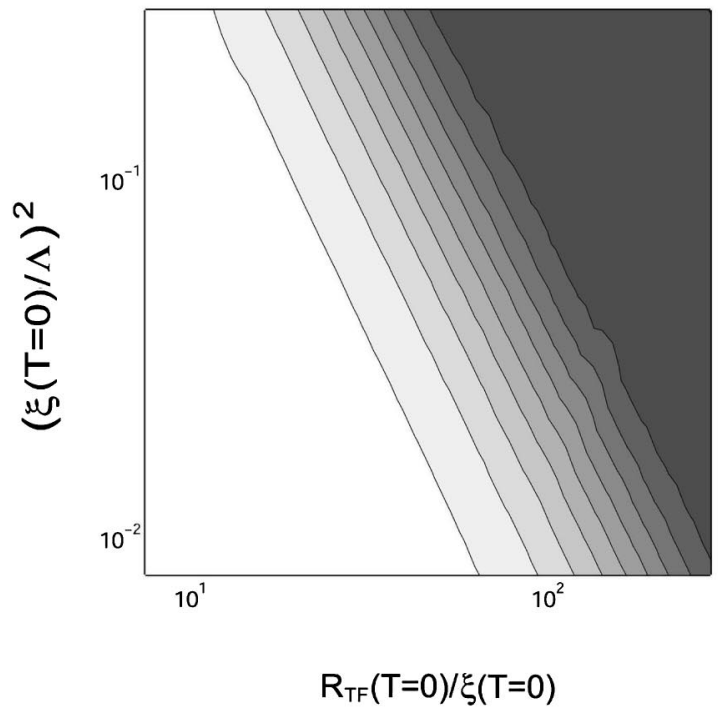

FIG. 6. Contour plot corresponding to Fig. 5, with both axes plotted on (a) linear and (b) logarithmic scale. As in Fig. 4, each contour corresponds to a value of $g$ ranging from 0.1 (leftmost contour) to 0.9 (rightmost contour). White regions correspond to true Bose-Einstein condensation, and darkest regions to the absence of phase coherence over the system size. Note that gray scale in this figure is not the same as in Fig. 5. Note also that we have not explicitly computed for $R_{\mathrm{TF}}(T=0) / \xi(T=0)<7$, where there is essentially true Bose-Einstein condensation, as this requires extremely high temperatures and large longitudinal trapping frequencies. The condition of validity of the Thomas-Fermi approximation will also break down in this limit.

part of the top left of Fig. 6(a), which requires the experiments to be performed at sufficiently low temperatures (here $T \ll 240 \mathrm{nK})$. We see that for low enough temperatures and small enough atom numbers, it is possible to probe experimentally the entire phase coherence regime (from $g=0$ to roughly $g=0.99$ ). Although the coherence decreases with (a)

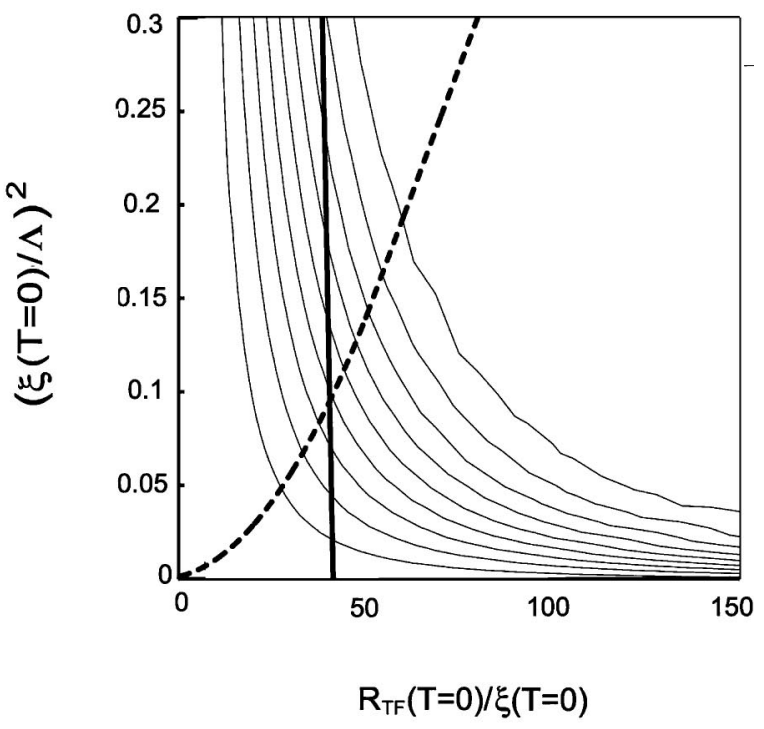

(b)

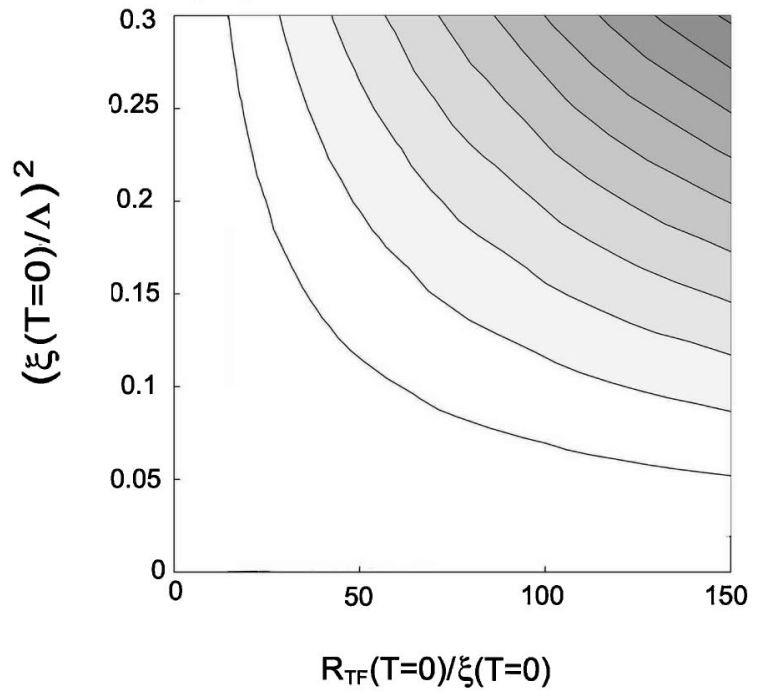

FIG. 7. (a) Same contour plot as in Fig. 6(a) indicating clearly the regions experimentally accessible for $N=2000{ }^{23} \mathrm{Na}$ atoms with $\omega_{\perp} / 2 \pi=5 \mathrm{KHz}$. The solid line corresponds to the condition $\mu$ $=\hbar \omega_{\perp}$, whereas the dashed line indicates the regime where $k_{B} T$ $=\hbar \omega_{\perp}$. Experimentally accessible regions due to actual quasi-1D constraints are located to the right of each of these lines. (b) Corresponding contour plot of quasicondensate fraction. Each contour corresponds to a change in $N_{0} / N$ of 0.05 with the leftmost contour indicating the region where $N_{0} / N=0.95$ and the rightmost contour in the plotted region corresponding to $N_{0} / N=0.5$. It is thus evident that the quasicondensate depletion becomes significant very rapidly as one moves away from the origin of the figure.

increasing scattering length, for example by tuning around a Feshbach resonance $[29,30]$, or central density, this can further restrict the region of accessibility due to the chemical potential constraint.

Figure 7(b) gives the corresponding contour plot of the (quasi)condensate fraction. It is evident that the (quasi)condensate depletion becomes important even within the range 
of this figure, with the furthermost points here corresponding to more than $50 \%$ depletion. In our analysis, we have chosen to monitor the loss of phase coherence by looking at the amount of decrease of the normalized two-point correlation function near the edge of the system [at $\left.z=0.9 R_{\mathrm{TF}}(T)\right]$. However, it may be experimentally easier to look at the decay of this correlation function at a point somewhat closer to the center. This would then lead to a slower decay of phase coherence across the measured distance, and a sufficiently small amount of phase coherence across such a region would then occur for even larger (quasi)condensate depletions. Therefore, we believe that a quantitative study of the phasecoherence crossover problem requires a theory which takes into account this depletion. In the regime where depletion can be neglected, the theory of Petrov et al. is of course also valid.

\section{CONCLUSIONS}

We have obtained a detailed universal picture of the coherence properties of a Bose gas as a function of the dimensionality, the temperature, and the interaction strength, paying particular attention to the crossover from quasicondensation to true Bose-Einstein condensation. In a finite homogeneous system at zero temperature, the long-range coherence is lost when the dimension of the system is reduced to one. This dimensional reduction leads to the appearance of a quasicondensate, whose coherence extends over a range that is much larger than the coherence length but much smaller than the size of the system. Similar results were obtained when the effects of temperature and the strength of the mean-field interaction were investigated. In a homogeneous one-dimensional system, a true condensate is located in a region in the phase diagram where both the length of the system and its temperature are small. Away from this region, a quasicondensate is present covering a wider region of the phase diagram.

A similar phase diagram was obtained for a harmonically confined system in one dimension, where a change in the confining potential at fixed temperature leads to a change in the size of the (quasi)condensate. It is important to note that our theory covers the entire temperature range, from zero temperature up to the crossover to the normal phase, where the (quasi)condensate becomes fully depleted. Since our work takes the (quasi)condensate depletion into account, the presented results extend beyond existing treatments, which have been limited to the regime of negligible depletion $[4,5,16]$. Note also that the phase diagram presented in Sec. IV corresponds to the pure one-dimensional limit. Nonetheless, a large part of this, ranging from complete absence of to complete coherence over the system, can be probed experimentally with existing technology, as discussed by a particular example based on typical parameters.

\section{ACKNOWLEDGMENTS}

This work was supported by the Stichting voor Fundamenteel Onderzoek der Materie (FOM), which is supported by the Nederlandse Organisatie voor Wetenschappelijk Onderzoek (NWO). N.P.P. acknowledges funding from the U.K. EPSRC and the hospitality of the ITP.
[1] H. Shi and A. Griffin, Phys. Rep. 304, 1 (1998); J.O. Andersen, e-print cond-mat/0305138.

[2] W.J. Mullin, J. Low Temp. Phys. 106, 615 (1997).

[3] T.-L. Ho and M. Ma, J. Low Temp. Phys. 115, 61 (1999).

[4] D.S. Petrov, G.V. Shlyapnikov, and J.T.M. Walraven, Phys. Rev. Lett. 85, 3745 (2000).

[5] D.S. Petrov, M. Holzmann, and G.V. Shlyapnikov, Phys. Rev. Lett. 84, 2551 (2000).

[6] N.D. Mermin and H. Wagner, Phys. Rev. Lett. 22, 1133 (1966).

[7] P.C. Hohenberg, Phys. Rev. 158, 383 (1967).

[8] J.O. Andersen, U. Al Khawaja, and H.T.C. Stoof, Phys. Rev. Lett. 88, 070407 (2002).

[9] U. Al Khawaja, J.O. Andersen, N.P. Proukakis, and H.T.C. Stoof, Phys. Rev. A 66, 013615 (2002); 66, 059902(E) (2002).

[10] A. Görlitz, J.M. Vogels, A.E. Leanhardt, C. Raman, T.L. Gustavson, J.R. Abo-Shaeer, A.P. Chikkatur, S. Gupta, S. Inouye, T.P. Rosenband, D.E. Pritchard, and W. Ketterle, Phys. Rev. Lett. 87, 130402 (2001).

[11] F. Schreck, L. Khaykovich, K.L. Corwin, G. Ferrari, T. Bourdel, J. Cobizolles, and C. Salomon, Phys. Rev. Lett. 87, 080403 (2001).

[12] H. Ott, J. Fortagh, G. Schlotterbeck, A. Grossmann, and C. Zimmermann, Phys. Rev. Lett. 87, 230401 (2001).

[13] W. Hänsel, P. Hommelhoff, T.W. Hänsch, and J. Reichel, Na- ture (London) 413, 501 (2001).

[14] S. Schneider, A. Kasper, Ch. vom Hagen, M. Bartenstein, B. Engeser, T. Schumm, I. Bar-Joseph, R. Folman, L. Feenstra, and J. Schmiedmayer, Phys. Rev. A 67, 023612 (2003).

[15] V.N. Popov, Theor. Math. Phys. 11, 565 (1972); Functional Integrals in Quantum Field Theory and Statistical Physics (Reidel, Dordrecht, 1983), Chap. 6.

[16] D.S. Petrov, G.V. Shlyapnikov, and J.T.M. Walraven, Phys. Rev. Lett. 87, 050404 (2001).

[17] S. Dettmer, D. Hellweg, P. Ryytty, J.J. Arlt, W. Ertmer, K. Sengstock, D.S. Petrov, G.V. Shlyapnikov, H. Kreutzmann, L. Santos, and M. Lewenstein, Phys. Rev. Lett. 87, 160406 (2001).

[18] F. Gerbier, J.H. Thywissen, S. Richard, M. Hugbart, P. Bouyer, and A. Aspect, Phys. Rev. A (to be published); e-print cond-mat/0211094.

[19] S. Richard, F. Gerbier, J.H. Thywissen, M. Hugbart, P. Bouyer, and A. Aspect, e-print cond-mat/0303137.

[20] D. Hellweg, L. Cacciapuoti, M. Kottke, T. Schulte, K. Sengstock, W. Ertmer, and J.J. Arlt, e-print cond-mat/0303308.

[21] I. Shvarchuck, Ch. Buggle, D.S. Petrov, K. Dieckmann, M. Zielonkowski, M. Kemmann, T.G. Tiecke, W. von Klitzing, G.V. Shlyapnikov, and J.T.M. Walraven, Phys. Rev. Lett. 89, 270404 (2002).

[22] M. Schick, Phys. Rev. A 3, 1067 (1971). 
[23] D.S. Fisher and P.C. Hohenberg, Phys. Rev. B 37, 4936 (1988).

[24] F.D.M. Haldane, Phys. Rev. Lett. 47, 1840 (1981).

[25] L. Tonks, Phys. Rev. 50, 955 (1936).

[26] M. Girardeau, J. Math. Phys. 1, 516 (1960).

[27] M. Olshanii, Phys. Rev. Lett. 81, 938 (1998).

[28] K. Bongs, S. Burger, S. Dettmer, D. Hellweg, J. Arlt, W. Ert- mer, and K. Sengstock, Phys. Rev. A 63, 031602(R) (2001).

[29] S. Inouye, M.R. Andrews, J. Stenger, H.-J. Miesner, D.M. Stamper-Kurn, and W. Ketterle, Nature (London) 392, 151 (1998).

[30] S.L. Cornish, N.R. Claussen, J.L. Roberts, E.A. Cornell, and C.E. Wieman, Phys. Rev. Lett. 85, 1795 (2000). 